\title{
BIOMIMETISMO APLICADO AO DESIGN SUSTENTÁVEL NO AMBIENTE CONSTRUÍDO - Uma Revisão Bibliográfica Sistemática
}

\author{
Maytê Galvão Pereira de Camargo \\ UFPR \\ maytecamargo@gmail.com
Alexandre Vieira Pelegrini
UTFPR
avpelegrini@yahoo.com

Resumo: O presente artigo explora os principais fundamentos e conceitos para a pesquisa relacionada ao tema biomimetismo aplicado ao design sustentável, com ênfase no ambiente construído. Para tanto, é realizada uma revisão bibliográfica sistemática (RBS) a respeito dos temas relacionados. Apresenta ainda, de forma introdutória, os conceitos de design sustentável e biomimetismo aplicado ao design, com exemplos de estudos de caso. Os resultados alcançados na RBS apontam para uma carência na literatura em relação ao assunto, porém, um crescente desenvolvimento nos últimos anos. A maioria significativa das pesquisas encontradas foram desenvolvidas na Europa e Estados Unidos. No Brasil, os estados com maior número de publicações são os da Região sul e Sudeste. Esta pesquisa possibilita o mapeamento do conhecimento atualizado na área e ainda identifica lacunas no tema. Portanto, instiga a continuação das pesquisas de forma organizada e sistematizada, com bibliografias préselecionadas e alguns dos conceitos definidos.

Palavras-chave: revisão bibliográfica sistemática, design sustentável, ambiente construído sustentável, biomimetismo no design.

\footnotetext{
Abstract: This article explores the key concepts and fundamentals for the research about biomimicry applied to sustainable design, with emphasis on the built environment. Therefore, a systematic literature review regarding issues related is performed. It further presents, in an introductory way, the concepts of sustainable design and biomimicry applied to design, with examples of case studies. The results achieved in the RBS point out the relative lack of literature on the subject, however, a growing development in recent years. A significant majority of the studies found were conducted in Europe and the United States. In Brazil, the states with the largest number of publications are those in the South and Southeast. This research provides updated knowledge in the area and also identifies gaps in the
} 
theme. Thereby, instigates further research in an organized and systematic way, with pre-selected bibliographies and some of the concepts defined.

Keywords: systematic literature review, sustainable design, built environment, biomimicry in design.

\section{INTRODUÇÃO}

O planeta Terra recebe 80 milhões de novos habitantes por ano, pressionando os sistemas naturais e causando vários impactos. Em 1930, o número de habitantes chegou a dois bilhões e, a partir deste momento, obteve-se um aceleramento a um ritmo desenfreado, acrescentando mais 5 bilhões de novos habitantes/consumidores em apenas oito décadas (WORLDWATCH, 2013).

Isto fez com que as áreas ficassem cada vez mais populosas e povoadas. Entretanto, muitas vezes sem planejamento adequado e com consumo desenfreado, que segundo Silva (2012) é um dos resultados da globalização, o "superconsumo". Portanto, é necessário o desenvolvimento de sistemas que propiciem uma maior eficiência energética, um melhor aproveitamento de água (com a sua reutilização), uma maior reciclagem de materiais e menos desperdícios, além da utilização de materiais ecologicamente corretos, entre outras formas.

$\mathrm{Na}$ década de 50-60 iniciam as primeiras preocupações com as questões da sustentabilidade (SANTOS, 2009). Inicia-se a concepção de que caberia aos projetistas, designers, arquitetos e engenheiros, planejar um mundo mais sustentável, com uma abordagem mais holística da Natureza e de forma tecnologicamente orientada. Nasce então a ciência denominada Biomimética (SOARES, 2008), focada na adaptação dos princípios da natureza à tecnologia. A natureza pode servir de modelo e inspiração para inovação no design de produtos e de interiores.

Segundo palestra, realizada em 2013 por Michael Pawlyn em Copenhague, "Se pudéssemos aprender a fazer e realizar coisas da mesma maneira que a natureza faz, nós poderíamos realmente atingir melhoras radicais na eficiência dos recursos" (PAWLYN, 2014, tradução nossa). Para um futuro positivo, o Biomimetismo é uma das mais importantes fontes de solução que permitirá o avanço da era industrial para a era ecológica (PAWLYN, 2014).

Janine Beynus, presidente do Biomimicry $3.8^{1}$, afirma que a ciência do biomimetismo não é nova; índios sempre dependeram das lições e exemplos da natureza ao seu redor. Os caçadores no Alaska ainda caçam focas da mesma forma que os ursos polares o fazem. Muitas invenções ocidentais, como o avião e o telefone, foram inspiradas na natureza (BIOMIMICRY 3.8, 2014).

No Biomimicry 3.8, nós acreditamos que não existe melhor parceiro de design do que a natureza. Mas biomimetismo é mais do que simplesmente olhar a forma de uma flor ou uma libélula e se inspirar; é uma metodologia que está sendo usada por algumas das maiores empresas e mais inovativas universidades no mundo (BIOMIMICRY 3.8, 2014, tradução nossa).

\footnotetext{
${ }^{1}$ Biomimicry 3.8 Institute - Organização sem fins lucrativos que promove o estudo do Biomimetismo, onde 3.8 é o número em bilhões de anos de evolução do universo - biomimicry.net
} 
Este artigo tem como objetivo, através de uma pesquisa inicialmente especulativa e depois sistemática, identificar os pilares de embasamento do estudo, principais conceitos, localizar os principais autores, livros e artigos sobre o assunto e identificar as lacunas existentes nas áreas, a fim de descobrir como aplicar os conceitos de biomimetismo na criação de produtos de design inovadores que propiciem ao ambiente construído a promoção da sustentabilidade.

Através das pesquisas, exploratória e da RBS, que é, resumidamente, uma revisão da literatura de forma organizada, é possível alcançar estes objetivos. Primeiramente encontram-se os principais artigos e autores, analisam-se os significados de design sustentável, biomimetismo, e este último aplicado ao design sustentável, bem como alguns exemplos aplicados como estudos de caso.

Os resultados alcançados na RBS apresentam, relativamente pouca literatura relacionada ao assunto, entretanto, um crescente desenvolvimento nos últimos anos, conforme será apresentado. Esta pesquisa possibilita conhecimento atualizado na área e ainda identifica a carência de trabalhos e produtos desenvolvidos.

\section{METODOLOGIA}

Para identificação dos principais autores e artigos, bem como os países com maior desenvolvimento do tema, é importante realizar uma revisão bibliográfica sistemática. Segundo Conforto et al (2011), uma RBS é constituída de 3 fases: Entrada, Processamento e Saída. Na fase de entrada é definido qual é o problema a ser respondido e os objetivos da pesquisa - ambos alocados na seção 1; as fontes primárias; os strings de busca; os critérios de inclusão e de qualificação; os métodos e ferramentas; e definido o cronograma de pesquisa (CONFORTO ET AL, 2011).

A primeira coleta e seleção de trabalhos para a RBS foi realizada entre março e abril de 2014 e possuiu os seguintes strings de busca, na língua inglesa: <biomimicry AND sustainability AND (design OR architecture)>, podendo variar de portal para portal de pesquisa.

As fontes primárias ou portais de pesquisa escolhidos foram: Portal da Capes ${ }^{2}$, ScienceDirect ${ }^{3}$ e Google acadêmico ${ }^{4}$. Os critérios de inclusão nos periódicos não excluíram qualquer material, não tiveram definições de datas ou língua e não são obrigatoriamente revisados por pares.

Após esta seleção prévia, que na Capes, por exemplo, resultou em 157 trabalhos, foram selecionados artigos, revisados por pares, desde 2003 e excluídos artigos em alemão e com tópicos não relacionados às áreas de Arquitetura e Design de Interiores. Como resultado ainda foram encontrados 64 trabalhos.

Então, definiu-se fazer uma segunda coleta de estudos, aumentando os strings de busca, mas ainda somente em inglês, para: <biomimicry AND sustainability AND (design OR architecture) AND "built environment" $>$, resultando em 21 trabalhos no Portal da Capes.

Assim, iniciou-se a fase 2, o processamento dos trabalhos encontrados. Executou-se a filtragem dos artigos conforme Conforto et al (2011) propõe. O filtro 01

\footnotetext{
${ }^{2}$ http://www.periodicos.capes.gov.br/

${ }^{3} \mathrm{http}: / /$ www.sciencedirect.com/

${ }^{4}$ http://scholar.google.com.br/
} 
(leitura de títulos, resumos e palavras-chave) e o 02 (leitura de introdução e conclusão) puderam ser inteiramente concluídos nesta primeira etapa; já o filtro 03 (leitura total dos textos) será realizado por completo na segunda fase de pesquisa, portanto realizado parcialmente neste momento.

Como saída, fase 3 de Conforto et al (2011), foi elaborada uma planilha em Excel com todos os trabalhos selecionados para a possível análise e conclusão e maior organização das informações encontradas, com identificação daqueles que disponibilizam ou não arquivos em pdf, de maneira gratuita ou paga, quando possível o país de desenvolvimento da pesquisa, o ano e o periódico da publicação, quais filtros foram realizados até o momento, além de outras informações pertinentes.

Quadro 1 - Exemplo do arquivamento no Excel feito pela RBS após conclusão dos strings de busca.

\begin{tabular}{|c|c|c|c|c|c|c|c|c|c|c|c|}
\hline \multirow[t]{2}{*}{$\mathbf{N} \underline{0}$} & \multirow[t]{2}{*}{ PDF } & \multirow{2}{*}{$\begin{array}{l}\text { OPEN } \\
\text { access }\end{array}$} & \multirow[t]{2}{*}{ TÍTULO } & \multirow[t]{2}{*}{ AUTOR } & \multirow[t]{2}{*}{ PERIÓDICO } & \multirow{2}{*}{$\begin{array}{l}\text { ANO } \\
\text { PUB. }\end{array}$} & \multirow[t]{2}{*}{ PAÍS } & \multicolumn{3}{|c|}{ FILTROS } & \multirow[t]{2}{*}{ OBS. } \\
\hline & & & & & & & & 01 & 02 & 03 & \\
\hline 1 & Sim & Sim & $\begin{array}{c}\text { Biomimicry as a } \\
\text { problem solving } \\
\text { methodology in } \\
\text { interior } \\
\text { architecture }\end{array}$ & $\begin{array}{c}\text { Rasha } \\
\text { Mahmoud } \\
\text { Ali El-Zeiny }\end{array}$ & $\begin{array}{l}\text { Procedia- } \\
\text { Social and } \\
\text { Behavioral } \\
\text { Sciences }\end{array}$ & 2012 & Egito & $x$ & $x$ & $x$ & \\
\hline
\end{tabular}

Fonte: Elaborado pelo autor, com base na pesquisa realizada.

Outro objetivo desta pesquisa é avaliar o desenvolvimento anual do tema. Para tanto, a partir do Google acadêmico, delimitando datas nas definições de busca, podese comparar a quantidade de publicações encontradas ano a ano sobre o assunto.

Além dos artigos pesquisados na RBS, duas das principais referências na internet a esse respeito podem ser encontradas em Asknatur ${ }^{5}$ e Biomimicry $3.8^{6}$. Nestes portais são pesquisados alguns conceitos e também produtos já existentes inspirados na natureza que atuem de forma sustentável.

\section{RESULTADOS}

A análise do desenvolvimento anual do assunto foi feita através do Google acadêmico, com o string de busca "biomimicry and design", conforme demonstrado no quadro 2:

\footnotetext{
${ }^{5}$ http://www.asknature.org/

${ }^{6}$ biomimicry.net
} 
Quadro 2 - Resultados comparativos anuais

\begin{tabular}{|c|c|}
\hline DATA & $\begin{array}{c}\text { QUANTIDADE TRABALHOS } \\
\text { PUBLICADOS }\end{array}$ \\
\hline Até abril 2014 & 366 \\
\hline 2013 & 1234 \\
\hline 2012 & 1000 \\
\hline 2011 & 600 \\
\hline 2010 & 330 \\
\hline 2009 & 1840 \\
\hline $2004-2009$ & 1670 \\
\hline $2000-2004$ & 410 \\
\hline Antes 2000 & 158 \\
\hline
\end{tabular}

Fonte: Elaborado pelo autor, com base na pesquisa realizada.

O quadro 2 permite concluir que há um crescimento em evolução nas pesquisas. Antes do ano 2000 (aproximadamente 40 anos de desenvolvimento da ciência do biomimetismo) havia pouquíssimos estudos. Nos 4 anos seguintes foram desenvolvidos 410 trabalhos, e de 2004 a 2009, este número ampliou-se para 1670. Em 2009, mesmo ano do lançamento do livro ${ }^{7}$ de Janine Benyus sobre 0 assunto, obteve-se um incrível salto com a publicação de 1840 estudos em um único ano. Com base na análise do quadro, é possível perceber que a partir do ano de 2010, a "febre" baixou, mas o aumento anual é visível devido à importância do tema.

Os resultados encontrados nos diferentes portais de pesquisa com os strings de buscas definidos na metodologia podem ser visualizados no quadro 3 :

Quadro 3 - Resultados encontrados nos diferentes portais.

\begin{tabular}{|c|c|c|c|c|}
\hline PORTAL & STRINGS & EXCLUSÕES & $\begin{array}{c}\text { QUANT. } \\
\text { PRÉVIA }\end{array}$ & $\begin{array}{c}\text { APÓS } \\
\text { FILTROS }\end{array}$ \\
\hline CAPES & $\begin{array}{c}\text { biomimicry AND (design OR } \\
\text { architecture) AND sustainability }\end{array}$ & $\begin{array}{c}\text { Somente artigos, revisados por pares, } \\
\text { desde 2003 e excluídos - alemão e } \\
\text { com tópicos não relacionados. }\end{array}$ & 64 & - \\
\hline CAPES & $\begin{array}{c}\text { biomimicry AND (design OR } \\
\text { architecture) AND sustainability } \\
\text { AND "built environment” }\end{array}$ & - & 21 & 20 \\
\hline $\begin{array}{c}\text { Science } \\
\text { Direct }\end{array}$ & $\begin{array}{c}\text { biomimicry AND (design OR } \\
\text { architecture) AND sustainability } \\
\text { AND "built environment” }\end{array}$ & - & 26 & 18 \\
\hline $\begin{array}{c}\text { Google } \\
\text { Acadêmico }\end{array}$ & $\begin{array}{c}\text { biomimicry AND (design OR } \\
\text { architecture) AND sustainability } \\
\text { AND "built environment” }\end{array}$ & Em português & 702 & - \\
\hline $\begin{array}{c}\text { Google } \\
\text { Acadêmico }\end{array}$ & $\begin{array}{c}\text { sustentabilidade AND (design OR } \\
\text { arquitetura) }\end{array}$ & & 64 & 15 \\
\hline
\end{tabular}

Fonte: Elaborado pelo autor, com base na pesquisa realizada.

A partir destes trabalhos selecionados na RBS e mais alguns encontrados de forma exploratória, foi possível criar um quadro comparativo dos principais países e autores pesquisadores, dentro da área de biomimetismo aplicado ao design de interiores e/ou arquitetura, apresentado no quadro 4:

\footnotetext{
${ }^{7}$ Biomimética - Inovação Inspirada pela Natureza
} 
Quadro 4 - Resultados relativos aos países dos autores nas publicações

\begin{tabular}{|c|c|c|c|}
\hline $\begin{array}{c}\text { PAÍS / } \\
\text { CONTINENTES }\end{array}$ & & \multicolumn{2}{|c|}{$\begin{array}{l}\text { QUANTIDADE ARTIGOS PRINCIPAIS } \\
\text { PUBLICADOS SELECIONADOS }\end{array}$} \\
\hline \multirow{5}{*}{ Brasil } & Paraná & 4 & \multirow{5}{*}{11} \\
\hline & Santa Catarina & 3 & \\
\hline & Rio Grande do Sul & 2 & \\
\hline & São Paulo & 1 & \\
\hline & Rio de Janeiro & 1 & \\
\hline \multirow[b]{3}{*}{ Europa } & Reino Unido & 7 & \multirow[b]{3}{*}{24} \\
\hline & Portugal & 5 & \\
\hline & $\begin{array}{c}\text { Demais países (Itália, Alemanha, Grécia, } \\
\text { Moldávia, Grécia, Romênia, Áustria, } \\
\text { Espanha, Holanda, Turquia, Suíça) }\end{array}$ & 12 & \\
\hline África & & \multicolumn{2}{|c|}{2} \\
\hline Ásia & & \multicolumn{2}{|c|}{4} \\
\hline Oceania & & \multicolumn{2}{|c|}{6} \\
\hline EUA & & \multicolumn{2}{|c|}{11} \\
\hline Canadá & & \multicolumn{2}{|c|}{3} \\
\hline $\begin{array}{l}\text { América } \\
\text { Central }\end{array}$ & & \multicolumn{2}{|c|}{1} \\
\hline
\end{tabular}

Fonte: Elaborado pelo autor, com base na pesquisa realizada.

O quadro 4 possibilita constatar que o tema está sendo desenvolvido em todos os continentes, mesmo que ainda em baixa quantidade, e a maioria dos autores mais relevantes estão localizados nos Estados Unidos (localizado o principal Instituto de pesquisa da área, com sede em Montana) e Reino Unido, além da Europa como um todo. Em Portugal existe também um desenvolvimento reconhecido, assim como no Brasil, que apresenta pesquisadores interessados no assunto, em Universidades, Congressos e Eventos realizados especialmente nos estados do Sul e Sudeste do país.

\section{DISCUSSÃO}

Após análise dos resultados obtidos, conclui-se que embora em crescimento, as pesquisas ainda necessitam ser ampliadas, mormente na área do design, para responder com eficácia as questões impostas pelas demandas de sustentabilidade mundial. Segundo Rocha Loures:

\footnotetext{
Sustentabilidade vem sendo utilizada como ponto de partida para a construção de um novo modelo de sociedade, capaz de garantir a sobrevivência dos seres humanos e da natureza. O recente histórico de consolidação do conceito faz com que este ainda seja representado por noções um pouco genéricas e difusas. Parte-se da premissa fundamental do reconhecimento da insustentabilidade dos padrões de desenvolvimento contemporâneos, da finitude dos recursos naturais e da manutenção das injustiças sociais (ROCHA LOURES, 2009, p. 59).
}

Isto justifica um estudo mais aprofundado do biomimetismo aplicado ao design. Com base na RBS, são apresentados alguns dos conceitos relativos ao tema.

\subsection{Conceito de design sustentável}

Um produto, sistema ou serviço deve apresentar os três pilares da sustentabilidade (Econômico, Ambiental e Social) para que seja considerado 
sustentável. A Organisation for Economic Co-operation and Development (OECD, 2002) identificou 5 objetivos das Edificações sustentáveis: Eficiência dos recursos; eficiência energética (incluindo redução na emissão de gases); Prevenção de poluição (incluindo qualidade do ar interno e redução de ruídos); harmonização com o ambiente; e abordagens integradas e sistêmicas.

Segundo Vezzoli (2008), o design para a sustentabilidade capacita o sistema de produção para responder à demanda social do bem-estar, utilizando drasticamente menos quantidades de recursos naturais do que os sistemas não sustentáveis. Isto exige administração coordenada de todas as ferramentas disponíveis, e promove a homogeneidade e visibilidade para todos os produtos, serviços e comunicação propostos.

O design ecológico é onde a variável ambiental é aplicada desde a concepção até o fim do ciclo de vida de um produto. E esta aplicação é uma tendência emergente, essencial para as empresas que desejam competir no mercado de trabalho. Soares (2008) também coloca que os impactos ambientais de um produto ocorrem em todas as fases do seu ciclo de vida, no entanto, a maioria é intrínseca às características do produto na fase de design, quando são tomadas as decisões mais críticas, em relação a custos, aparência, seleção de materiais, inovação, desempenho, longevidade, durabilidade e capacidade de reparação (SOARES, 2008).

\subsection{Conceito de biomimetismo}

Segundo El-Zeiny (2012), o campo do biomimetismo é onde flora, fauna ou ecossistemas inteiros são imitados como base de design para criar um ambiente construído mais sustentável. É uma ciência que produz soluções inovadoras para os problemas humanos através do estudo de designs naturais, processos e sistemas.

No entanto, a aplicação prática do método continua não sendo realizada. A arquitetura de interiores utiliza muito a biologia como fonte de inspiração de formas ou decoração, mas simplesmente imitar ou se inspirar em formas naturais, texturas e cores somente não é biomimetizar. Tem que possuir a ciência (além da forma, processos e ecossistemas) da biologia, não apenas a aparência (EL-ZEINY, 2012).

Abaixo se encontra um exemplo de imitação da natureza, inspirada em concha do mar. Ela também possui conceitos de sustentabilidade devido à resistência a terremotos e baixa manutenção, mas ainda não se encaixa nas definições de biomimetismo.
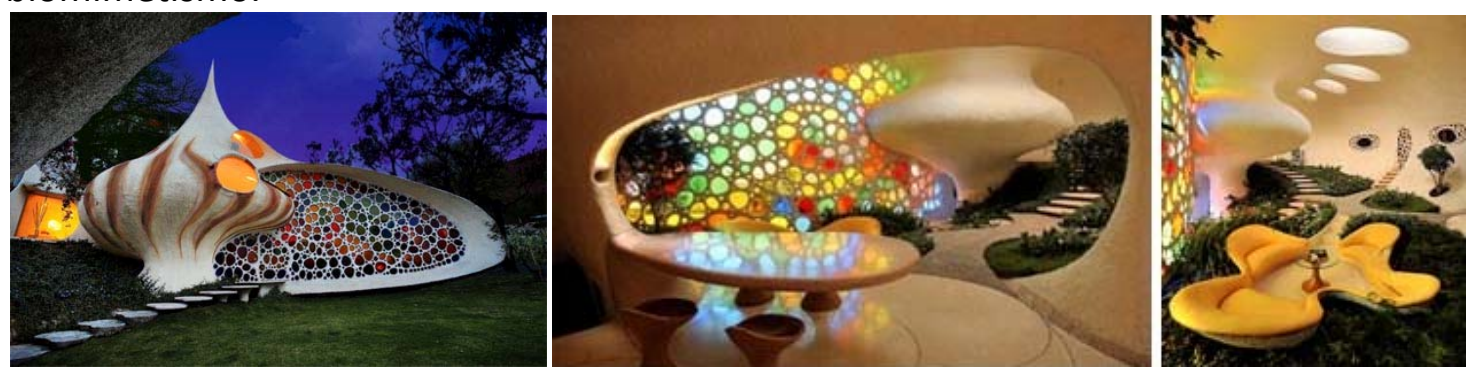

Figura 1 - A spiral Shell house, projetada por Senosiain Arquitetos.

Fonte: El-Zeiny, 2012

Segundo Gruber et al (2007) a natureza aperfeiçoou seu design durante bilhões de anos de evolução, então imitar suas criações, é um meio seguro de produzir tecnologias que sejam eficientes e confiáveis. E segundo John et al (200 5) a natureza 
contém materiais e estruturas mais benignos do que os itens produzidos pela indústria.

Resumindo outros autores, Soares (2008) afirma que nos sistemas naturais não existem resíduos, todos os materiais são reutilizados, geralmente com grande eficiência, os resíduos de uma espécie são o alimento de outra.

El-Zeiny (2012) apresenta em seu trabalho um quadro com as principais características que podem ser "mimetizadas" da natureza, que podem ser características do próprio organismo - como forma, cor, movimento; podem ser através de suas relações com a comunidade - como interação com outros organismos ou administração e técnicas de sobrevivência; o suas relações com o bioma em que vive - como camuflagem, refrigeração . O quadro 5 resume as principais.

Quadro 5 - Alguns dos principais níveis de informação para o biomimetismo

\begin{tabular}{|c|c|}
\hline Níveis de biomimetismo & Aspectos do Nível \\
\hline \multirow{8}{*}{ Recursos do organismo } & Forma, cor, volumetria, transparência, ritmo. \\
\hline & Estrutura, estabilidade e resistência à gravidade. \\
\hline & Mutação, crescimento e ciclo de vida. \\
\hline & Função e comportamento. \\
\hline & Movimento e aerodinâmica. \\
\hline & Auto-montagem. \\
\hline & Homeostase (equilíbrio). \\
\hline & $\begin{array}{l}\text { Sistemas orgânicos, digestivos, circulatórios, respiratórios, } \\
\text { sustentação, musculares, nervosos, excretórios, sensoriais e } \\
\text { de locomoção. }\end{array}$ \\
\hline \multirow{5}{*}{$\begin{array}{c}\text { Relação do organismo com a } \\
\text { comunidade }\end{array}$} & Técnicas de sobrevivência. \\
\hline & Interação com outras criaturas. \\
\hline & Transferência de conhecimento e treinamento. \\
\hline & Hierarquia dos membros. \\
\hline & Administração e coordenação do grupo. \\
\hline \multirow{6}{*}{$\begin{array}{c}\text { Relação do organismo com o } \\
\text { ambiente e bioma }\end{array}$} & Resposta ao clima por refrigeração, calefação e ventilação. \\
\hline & Resposta por camuflagem, auto-proteção e auto-limpeza. \\
\hline & Adaptação a diferentes níveis de luz, som, sombra. \\
\hline & Construção de abrigo. \\
\hline & Adaptação à limitada oferta de recursos. \\
\hline & Administração de resíduos. \\
\hline
\end{tabular}

Fonte: Elaborado pelo autor, com base na pesquisada realizada por El-Zeiny , 2012.

Segundo Biomimicry (2014), em entrevista com Janine Benyus, alguns dos pioneiros no estudo do biomimestimo, ligados à área de Design ou Arquitetura, são os listados no quadro abaixo, que representa também qual a área de pesquisa atual e o que estão desenvolvendo. 
Quadro 6 - Alguns dos pioneiros no estudo de biomimetismo relacionados ao design e o que estão fazendo.

\begin{tabular}{|c|c|c|}
\hline Pesquisador & \begin{tabular}{ll|} 
Universidade & / \\
Instituto & / \\
Laboratório &
\end{tabular} & Pesquisa \\
\hline $\begin{array}{l}\text { Thomas e Ana } \\
\text { Moore e Devins } \\
\text { Gust }\end{array}$ & University of Arizona & $\begin{array}{l}\text { Estudo de como a folha captura energia para criar célula } \\
\text { solar de tamanho molecular. }\end{array}$ \\
\hline J. Herbert Waite & $\begin{array}{lr}\text { University } & \text { of } \\
\text { California } & \text { Santa } \\
\text { Barbara } & \end{array}$ & $\begin{array}{l}\text { Estudo do mexilhão que se gruda nas rochas via um } \\
\text { adesivo para criar uma cola resistente e funcional sob a } \\
\text { água. }\end{array}$ \\
\hline Jay Harman & PAXscientific & $\begin{array}{l}\text { Criação de uma super eficiente pá de ventilador, } \\
\text { aeradores e hélices baseados na geometria do espiral } \\
\text { encontrado em conchas, kelp (tipo de alga) e chifres de } \\
\text { carneiros. }\end{array}$ \\
\hline A.K. Geim & $\begin{array}{l}\text { University } \\
\text { Manchester }\end{array}$ & $\begin{array}{l}\text { Desenvolveu processo sem cola baseado nas "cerdas" } \\
\text { da lagartixa, proporcionando desmontagem sem } \\
\text { contaminação. }\end{array}$ \\
\hline
\end{tabular}

Fonte: Elaborado pelo autor, com base na pesquisa realizada no site Biomimicry 3.8.

\subsection{Biomimetismo aplicado ao design sustentável}

Segundo Pandremenos et al (2012), o método da biomimetização pode ser aplicado de duas maneiras distintas. A primeira seria biologia - design, onde um fenômeno biológico contempla uma solução de design - Solution based approach; ou design - biologia, onde o inovador inicia a pesquisa com o problema no design, e depois revisa quais e como os organismos estão atingindo aquele objetivo - Problem based approach (El-Zeiny, 2012).

Faludi (2005), desenvolvendo a forma Problem based approach, afirma que para criar produtos biomiméticos deve-se seguir três passos:

- Definir o problema e o seu contexto

- Encontrar organismos com o mesmo problema e contexto. Avaliar o que fazem. Encontrar diversos exemplos e analisar qual tem a melhor e mais relevante estratégia

- Transformar a melhor estratégia em algo construtível. Se necessário, encontrar um expert para auxiliar.

No portal AskNature, encontram-se exemplos de estudos de caso aplicados como: Vidros imitando teias de aranha para prevenção de colisão de pássaros; Cola para madeira sem formaldeído utilizando a química azul do marisco; Carpet com fibra não direcionável e colorido; Energia wireless inspirada nas abelhas; Manutenção de qualidade de água em forma de espiral inspirada em diversas forma na natureza.

El-Zeiny (2012) também expõe em sua pesquisa estudos de caso de aplicação do biomimetismo na arquitetura de interiores, como o carpet, selecionado por ser o mais relacionado à esta pesquisa:

- Carpet (solution-based approach / nível dos recursos do organismo) inspirados pelo caos organizado de folhas caídas ou de pedriscos a beira do rio, descobriu-se que na natureza nunca havia as mesmas cores e conteúdos. 0 Entropy foi criado, inspirado pelos padrões aleatórios do chão da floresta, alcançando vantagens ambientais em comparação aos produtos atuais, por ser de mais fácil substituição (não necessita a combinação perfeita), possuir menos 
descartes, de mais fácil instalação (em qualquer sentido) e com redução de desperdício.

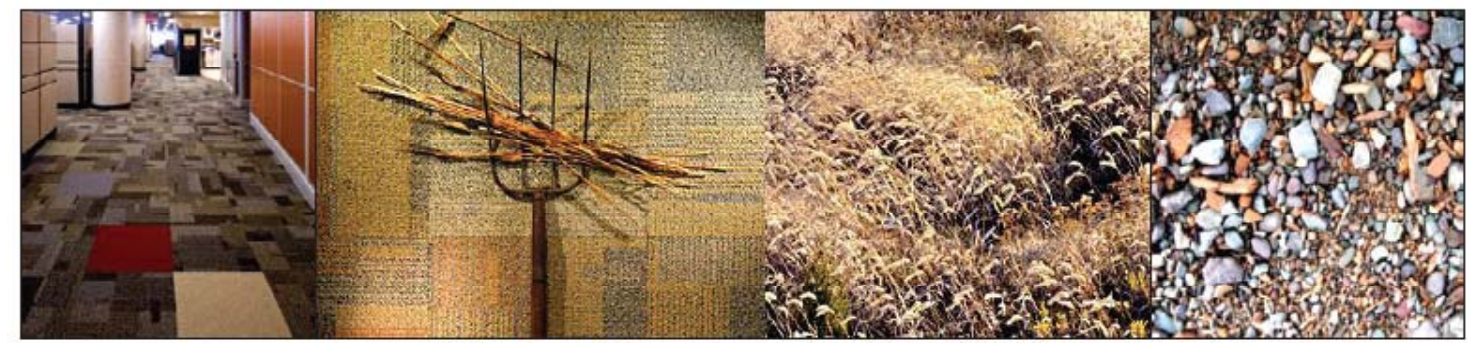

Figura 2 - Piso Entropy, imitando os padrões aleatórios no chão da floresta.

Fonte: El-Zeiny, 2012

Segundo Barba Neto (2013), um outro exemplo seria o piso antiderrapante, onde a fruta-do-conde serviu de inspiração para o design de superfícies, graças à morfologia de sua superfície e rugosidade.

Os estudos de caso enfatizam que se deve integrar os conceitos de biomimetismo já no início dos processos de design, antes mesmo das idéias preliminares, colocando como necessária a participação de um biólogo no desenvolvimento, um membro da equipe de pesquisa, e não apenas um consultor (ElZeiny, 2012). Todos os estudos obtiveram resultados sustentáveis e soluções eficazes para os mais importantes problemas nos ambientes interiores, como luz do dia, conforto térmico, eficiência energética, durabilidade e produtividade.

\section{CONCLUSÃO}

Este artigo foi escrito com o objetivo de identificar os alicerces, encontrar os conceitos fundamentais e alguns dos mais importantes autores e trabalhos sobre 0 tema. Isto foi possível através da pesquisa realizada, de forma especulativa e RBS.

Percebeu-se também que poderiam ser encontrados um maior número de trabalhos com outros strings de busca como Biônica, Bioinspiração e Biomimética, mas ainda não aplicados neste artigo.

Apesar de o biomimetismo estar sendo mais pesquisado, ainda percebe-se a falta de aplicação pelos poucos estudos de caso possíveis de serem analisados (Faludi, 2005).

Os resultados alcançados permitem concluir, relativamente a outros ramos do design, pouca bibliografia, e, entretanto, um crescente desenvolvimento nos últimos anos. Na bibliografia pesquisada, pode-se encontrar conhecimento atualizado na área e perceber a falta de trabalhos e produtos de design desenvolvidos e ainda a necessidade de equipes multidisciplinares.

Após análise dos resultados obtidos, conclui-se que é necessária a ampliação dos estudos sobre a ciência do biomimetismo no Brasil e no mundo, principalmente biomimetismo aplicado ao design.

Segundo El-Zeiny (2012), para a expansão da biomimética, os estudos de arquitetura e design deveriam incluir este ramo da ciência, e Giurea (2014) propõe em seu trabalho um método didático para transposição de formas naturais na arquitetura. Ainda networks, workshops e eventos relacionados ao tema devem ocorrer para que haja também a transferência de conhecimento entre designers e biólogos. 
Como sugestão para trabalhos futuros, seria o estudo de aplicação em estudos de caso, no desenvolvimento de produtos biomiméticos e até mais pesquisas de modo geral.

\section{AGRADECIMENTOS}

Os autores agradecem o apoio financeiro da CAPES (Coordenação de Aperfeiçoamento de Pessoal de Nível Superior) pela bolsa de mestrado demanda social.

\section{REFERÊNCIAS}

BARBA NETO, João. Estudo de elemento da natureza para aplicação em design: biomimetização da estrutura de ninhos de cacicus haemorrhous. 2013. $194 \mathrm{f}$. Dissertação (Mestrado em Design) - Universidade Federal do Paraná, Programa de Pós-Graduação em Design.

Biomimicry 3.8. Disponível na internet por http em: http://biomimicry.net/. Acesso em: 28 abr. 2014.

CONFORTO, Edivandro Carlos; AMARAL, Daniel Capaldo; SILVA, Sérgio Luis da. Roteiro para Revisão Bibliográfica Sistemática: aplicação no desenvolvimento de produtos e gerenciamento de projetos. Porto Alegre: 8‥ CBGDP, 2011.

EL-ZEINY, Rasha Mahmoud Ali. Biomimicry as a Problem Solving Methodology in Interior Architecture. Procedia - Social and Behavioral Sciences, no 50, p. 502-512, julho 2012.

FALUDI, J. Biomimicry for Green Design (a how to). World Changing, 2005. Disponível na internet por http em: http://www.worldchanging.com/archives/003680.html. Acesso em 28 abr. 2014.

GIUREA, Diana. A Didactic Method for Transposing Natural Forms in Architecture. Procedia - Social and Behavioral Sciences, no 116, p. 3165-3168, 2014.

GRUBER, P. ; IMHOF, B. Transformation: Structure / space studies in bionics and space design. Acta Astronautica, no 60, p. 561-570, 2007.

JOHN, G; CLEMENTS-CROOME, D; JERONIMIDIS, G. Sustainable building solutions: a review of lessons from the natural world. Building and Environment, no 40, p. 319-328, 2005.

OECD. Design of Sustainable Building Policies: Scope for Improvement and Barriers. Organisation for Economic Co-operation and Development, 2002. Disponível na internet por http em: http://www.oecd.org/env/consumptioninnovation/oecdworkonsustainablebuildings.htm. Acesso em 29 abr. 2014.

PANDREMENOS, J.; VASILIADIS, E.; CHRYSSOLOURIS,G. Design Architectures in Biology. Procedia CIRP, no 3, p. 448-452, 2012. 
PAWLYN, Michael. Biomimicry in Architectural design. Presentation from Velux Daylight Symposium, 2013. Disponível na internet por http em: https://www.youtube.com/watch?v=wdoriWPaaDI. Acesso em 30 abr. 2014.

ROCHA LOURES, Rodrigo C. da. Sustentabilidade XXI: Educar e inovar sob uma nova consciência. São Paulo: Editora Gente, 2009.

SANTOS, Tacilla da Costa e Sá S. Organizações da Sociedade Civil e as Construções Teóricas Contemporâneas Acerca da Sustentabilidade. Cadernos de Gestão social, vol. 2 no 1, p. 105-120, 2009.

SILVA, Christian L.da et al. Inovação e sustentabilidade. Curitiba: Aymará Educação, 2012.

SOARES, Marina Arminda Ribeiro. Biomimetismo e Ecodesign: Desenvolvimento de uma ferramenta criativa de apoio ao design de produtos sustentáveis. 2008. 84f. Dissertação (Mestrado em Engenharia do Ambiente) - Universidade Nova de Lisboa, Faculdade de Ciências e Tecnologia.

VEZZOLI, Carlo; MANZINI, Ezio. Design for Environmental Sustainability. Milan: Springer, 2008.

WORLDWATCH INSTITUTE. Estado do mundo 2013: A sustentabilidade ainda é possível? Salvador, 2013. 